\title{
TBI Calculations of the Elastic Properties and Structural Phase Transformation in Novel Materials: Yttrium Nitride
}

\author{
S. Singh ${ }^{a, *}$, R. CHAUHAN ${ }^{b}$ AND A. GOUR ${ }^{c}$ \\ ${ }^{a}$ High Pressure Research Lab, Physics Dept., Barkatullah University, Bhopal-462001, India \\ ${ }^{b}$ Mittal Institute of Technology, Nabi Bagh, Bhopal-462038, India \\ ${ }^{c}$ Govt. Polytechnic College Physics Department, HARDA, Indore Road (M.P.), India
}

(Received July 1, 2009; in final form August 2, 2011)

\begin{abstract}
We have carried out high pressure theoretical structural studies of yttrium nitride to examine the phase transition phenomena from the $\mathrm{NaCl}$ structure to $\mathrm{CsCl}$ structure by using a three-body potential model. The phase transition pressure $(140 \mathrm{GPa})$ predicted by this approach is close to the phase transition pressure, predicted by others $(138 \mathrm{GPa})$. Yttrium nitride is a novel and less explored material. Under high pressure yttrium nitride goes through a sudden collapse in volume showing the first order phase transition. To understand the effect of pressure we studied bulk properties, elastic constants and their combination. The pressure volume equation of state provides meaningful signatures of physical and chemical phenomena under high pressure. Moreover we have successfully checked the stability criterion for this compound.
\end{abstract}

PACS: 64.70.K-, 62.20.de, 62.50.-p

\section{Introduction}

The transition metal compounds MX (M denotes a transition metal element and $\mathrm{X}$ denotes one of the non-metallic elements $\mathrm{C}, \mathrm{N}$ or $\mathrm{O}$ ) have attracted attention during last decade because of their high hardness, high melting point, wear and corrosion resistance [1-9]. The investigation of the behavior of transition metal compounds under high pressure is very important because of two reasons, namely the interests in an antiferromagnetic Mott type insulator in solid state physics and their roles in the interior of the earth [2]. The transition (T) metal (M) nitrides (N) are valuable for several technological applications. Because of their great strength and durability, their long established use is as protective coatings [3]. However, they also have interesting optical, electronic, catalytic and magnetic properties $[1,2]$. In the electronic industry they are important as electric contact, diffusion barriers, buffer layer etc. In the last few years there has been an increased awareness in scandium, yttrium and lanthanum nitrides due to phase transition and their semiconducting properties [4-9]. The exchange and correlation effects were treated using the generalized gradient approximation (GGA) in case of yttrium nitride (YN) by Stampfl et al. [8].

In contrast with traditional semiconductors the group IIIB transition metal nitrides is interstitial alloys and they could have a wide array of chemical compositions [10-13]. These materials crystallize in the rock-salt structure and they can be either metal or semiconductors. First principle calculations have been carried out by

* corresponding author; e-mail: drsadhna_in@rediffmail.com
Mancera et al. [6] in YN. Mancera shows a $\mathrm{B}_{1}-\mathrm{B}_{2}$ structural phase transition pressure $P_{t}$ in $\mathrm{YN}$ at $138 \mathrm{GPa}$. Although the $P_{t}$ predicated by Mancera is high, but it is in consistency with other TMC's which crystallize in rock-salt structure. Band structure and cohesive properties of $\mathrm{YN}$ in the $\mathrm{NaCl}$ structure carried out by Haglund et al. [14]. It is also reported, while studying YN having NaCl-type structure, that their parent metal has its ground state in hexagonal symmetry, that is why $\mathrm{YN}$ system shows the hexagonal cubic phase transition [14]. They calculated their bulk properties and the Debye temperature. Some other physicists also carried out study on the electronic structure and physical properties of YN [8]. According to them this material is semimetal within the local density approximation (LDA) [15]. Cohesive energy and band structure of $3 d$-transition metal carbides and nitrides having $\mathrm{NaCl}$-type structure using muffin tin orbital method has been done by Haglund et al. [14].

They have also shown that $\mathrm{YN}$ is indirect semiconductor with gaps of $1-2 \mathrm{eV}$. Therefore transition metal nitrides represent a novel group of semiconductor materials [13].

$\mathrm{YN}$ is presently one of the most important materials for hardness and corrosion resistant coating with non-toxic and non-stick nature. Currently, the interest is also developing within the microelectronic industry for the use of $\mathrm{YN}$ as an electrically conducting barrier. This compound shows hardness with high melting points so study of their resistance to pressure also becomes important. In the present paper, efforts have been made to study phase transition pressure and volume collapse using three-body potential model. The three-body interactions have been considered to arise from the charge transfer effects during the overlapping of electron shells of the adjacent 
ions [16]. This three-body potential model (TBPM) has been found to be successful in describing the phase transition, phonon dispersion, harmonic and anharmonic properties of ionic and semiconductor solids [16-24].

\section{Theory and method}

The application of pressure causes compression or decrease in volume leading to increased charge transfer due to the deformation of the overlapping electron shells of the adjacent ions in solids. These effects have been incorporated in the Gibbs free energy $G$ as a function of pressure $(P)$ and three-body interactions (TBI) [17], which are the most dominant among many-body contributions. Three-body interactions are of great importance at high pressure when the interionic separation decreases considerably and coordination number increases. The Gibbs free energies, at $T=0 \mathrm{~K}$ and pressure $(P)$, for the real $\left(\mathrm{B}_{1}\right)$ and hypothetical $\left(\mathrm{B}_{2}\right)$ phases are given by

$$
\begin{aligned}
& G \mathrm{~B}_{1}(r)=U \mathrm{~B}_{1}(r)+P V_{\mathrm{B} 1}, \\
& G \mathrm{~B}_{2}(r)=U \mathrm{~B}_{2}(r)+P V_{\mathrm{B} 2},
\end{aligned}
$$

where the first terms in the above equations represent the lattice energies for the $\mathrm{B}_{1}$ and $\mathrm{B}_{2}$ structures expressed as

$$
\begin{aligned}
& U \mathrm{~B}_{1}(r)=-\alpha_{\mathrm{M}} Z e^{2}(Z+12 f(r)) / r \\
& \quad+6 b \beta_{+-} \exp \left(\left(r_{+}+r_{-}-r\right) / \rho\right) \\
& \quad+6 b \beta_{++} \exp \left(\left(2 r_{+}-\sqrt{2} r\right) / \rho\right) \\
& \quad+6 b \beta_{--} \exp \left(\left(2 r_{-}-\sqrt{2} r\right) / \rho\right), \\
& U \mathrm{~B}_{2}\left(r^{\prime}\right)=-\alpha_{\mathrm{M}}^{\prime} Z e^{2}\left(Z+16 f\left(r^{\prime}\right)\right) / r^{\prime} \\
& \quad+8 b \beta_{+-} \exp \left(\left(r_{+}+r_{-}-r^{\prime}\right) / \rho\right) \\
& \quad+3 b \beta_{++} \exp \left(\left(2 r_{+}-1.154 r^{\prime}\right) \rho\right) \\
& +3 b \beta_{--} \exp \left(\left(2 r_{-}-1.154 r^{\prime}\right) / \rho\right) .
\end{aligned}
$$

Here, the first two terms are the long-range Coulomb and TBI energies [16]. The remaining terms correspond to the overlap repulsion represented by Hafemeister and Flygare (HF) potential and extended up to the first $(+-)$ and second $(++$ and --$)$ neighbour ions, $\alpha_{\mathrm{M}}\left(\alpha_{\mathrm{M}}^{\prime}\right)$ is the Madelung constant for $\mathrm{B}_{1}=1.7478\left(\mathrm{~B}_{2}=1.7629\right)$ phase, $\beta_{i j}(i j=+-)$ are the Pauling coefficients defined as $\beta_{i j}=1+Z_{i /} n_{i}+Z_{j /} n_{j}$ with $Z_{i}\left(Z_{j}\right)$ and $n_{i}\left(n_{j}\right)$ as the valence and the number of electrons of the $i(j)$-th ion. $Z e$ is the ionic charge with $e$ as the electronic charge, $b(\rho)$ are the hardness (range) parameters, $r\left(r^{\prime}\right)$ is the nearest neighbor separation of $\mathrm{B}_{1}\left(\mathrm{~B}_{2}\right)$ phase and $f(r)$ is three-body interaction parameter which may be conveniently expressed as $f(r)=f_{0} \exp (-r / \rho)[15,16]$ with $f_{0}$ as a constant. The $r_{+}$and $r_{-}$are the ionic radii of ions. The lattice energies given by Eqs. (3) and (4) contain only three model parameters $(b, \rho$ and $f(r))$ whose values have been determined from measured interionic separation and cohesive energy after choosing an appropriate value of $\rho$ [17]. To understand elastic properties of these transition metal compounds we have calculated second order elastic constants (SOEC), $C_{11} C_{12}$ and $C_{44}$, and their pressure derivatives at $0 \mathrm{~K}$, since these elastic constants are functions of first and second order derivatives of short range potential, so their calculation will provide knowledge about the effect of short range forces on these materials following Sharma et al. [18] and Singh et al. [19] and Shanker et al. [20, 21]. The expressions for SOECs are as follows:

$$
\begin{aligned}
& C_{11}=\left(e^{2} / 4\right) r^{4}\left[-5.112 Z(Z+12 f(r))+\mathrm{A}_{1}\right. \\
& \left.\quad+\left(\mathrm{A}_{2}+\mathrm{B}_{2}\right) / 2\right], \\
& \quad C_{12}=\left(e^{2} / 4 r^{4}\right)\left[0.226 Z(Z+12 f(r))-\mathrm{B}_{1}\right. \\
& \left.\quad+\left(\mathrm{A}_{2}-5 \mathrm{~B}_{2}\right) / 4\right], \\
& C_{44}=\left(e^{2} / 4 r^{4}\right)\left[2.556 Z(Z+12 f(r))+\mathrm{B}_{1}\right. \\
& \left.\left.\quad+\left(\mathrm{A}_{2}+3 \mathrm{~B}_{2}\right) / 4\right)\right]
\end{aligned}
$$

with

$$
\begin{aligned}
& \mathrm{A}_{1}=\left(8 r^{3} / e^{2}\right)\left[\left(b / \rho^{2}\right) \exp \left(\left(r_{+}+r_{-}-r\right) / \rho\right)\right]_{r=a} \\
& \mathrm{~B}_{1}=\left(8 r^{2} / e^{2}\right)\left[(-b / \rho r) \exp \left(\left(r_{+}+r_{-}-r\right) / \rho\right)\right]_{r=a} \\
& \mathrm{~A}_{2}=\left(16 r^{3} / e^{2}\right)\left[( b / \rho ^ { 2 } ) \left(1.75 \exp \left(\left(2 r_{+}-r\right) / \rho\right)\right.\right. \\
& \left.\quad+0.25 \exp \left(\left(2 r_{-}-r\right) / \rho\right)\right]_{r=\sqrt{2} a} \\
& \mathrm{~B}_{2}=\left(16 r^{2} / e^{2}\right)\left[( - b / \rho r ) \left(1.75 \exp \left(\left(2 r_{+}-r\right) / \rho\right)\right.\right. \\
& \left.\quad+0.25 \exp \left(\left(2 r_{-}-r\right) / \rho\right)\right]_{r=\sqrt{2} a} .
\end{aligned}
$$

The expressions of pressure derivatives $\mathrm{d} S / \mathrm{d} p, \mathrm{~d} B_{T} / \mathrm{d} p$ and $\mathrm{d} C_{44} / \mathrm{d} p$ are as follows:

$$
\begin{aligned}
& \mathrm{d} S / \mathrm{d} p=-(2 \Omega)^{-1}\left[23.682 Z(Z+12 f(r))+C_{1}\right. \\
& \quad+\left(C_{2}+6 A_{2}-6 B_{2}\right) / 2-50.0752 Z a \partial f / \partial r \\
& \left.\quad+13.9808 Z a^{2} \partial^{2} f / \partial r^{2}\right], \\
& \quad \mathrm{dB}_{T} / \mathrm{d} p=-(3 \Omega)^{-1}\left[13.980 Z(Z+12 f(r))+C_{1}\right. \\
& \quad-3 A_{1}+C_{2}-3 A_{2}-167.7648 Z a \partial f / \partial r \\
& \left.\quad+41.9420 Z a^{2} \partial^{2} f / \partial r^{2}\right], \\
& \mathrm{d} C_{44} / \mathrm{d} p=-(\Omega)^{-1}\left[11.389 Z(Z+12 f(r))+A_{1}\right. \\
& \quad-3 B_{1}+\left(C_{2}+2 A_{2}-10 B_{2}\right) / 2 \\
& \quad-44.6528 Z a \partial f / \partial r]
\end{aligned}
$$

with

$$
\begin{aligned}
& \Omega=-2.330 Z(Z+12 f(r))+\mathrm{A}_{1}+\mathrm{A}_{2} \\
& \quad+27.9612+Z a \partial f / \partial r, \\
& C_{1}=\mathrm{A}_{1}^{2} / \mathrm{B}_{1}, \quad C_{2}=\mathrm{A}_{2}^{2} / B_{2},
\end{aligned}
$$

and

$$
S=\left(C_{11}-C_{12}\right) / 2 \text { and } B_{T}=\left(C_{11}+2 C_{12}\right) / 3 .
$$

Above expressions for elastic constant, pressure derivatives of shear modulus, bulk modulus and tetragonal modulus are given in Eqs. (5)-(14) taken from [18-21].

\section{Results and discussion}

The input three model parameters namely range, hardness and three-body interaction parameter are well illustrated in Table I. We have computed the different values of interionic separation $r\left(\mathrm{~B}_{1}\right)$ and $r^{\prime}\left(\mathrm{B}_{2}\right)$ at different 
pressures using the technique of minimization of $G_{\mathrm{B} 1}(r)$ and $G_{\mathrm{B} 2}\left(r^{\prime}\right)$, respectively. We have evaluated the values of $G_{\mathrm{B} 1}(r)$ and $G_{\mathrm{B} 2}\left(r^{\prime}\right)$ at different pressures and the corresponding Gibbs free energy difference $\Delta G$ (= $\left.G_{\mathrm{B} 1}(r)-G_{\mathrm{B} 2}\left(r^{\prime}\right)\right)$ have been plotted against pressure and given in Fig. 1. The pressure at which $\Delta G$ approaches zero corresponds to the phase transition pressure $P_{t}$. The value of $P_{t}$ is compared with available computed data in Table II. It is clear from Fig. 1 that our approach has predicted correctly the relative stability of competitive crystal structure as the value of $\Delta U$ is positive at zero pressure, it is a required criterion for the stability at parent phase [24]. This figure shows that the values of the phase transition pressure $\left(P_{\mathrm{t}}\right)$ obtained from the present model is closer to available predicted data by the first principle calculation method [6].

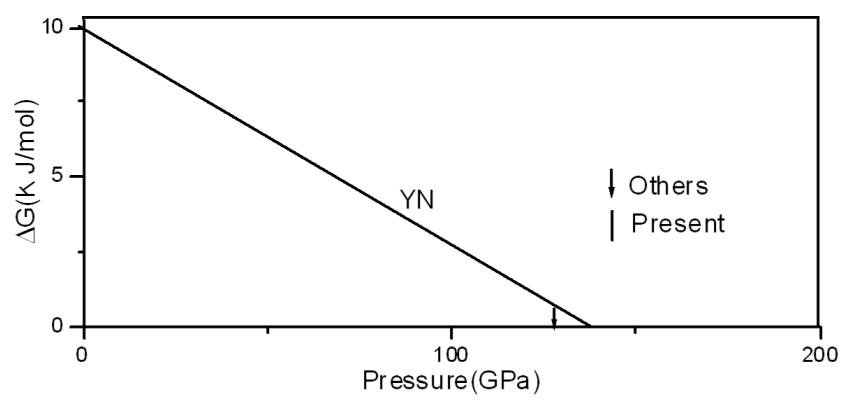

Fig. 1. Variation of Gibbs free energy differences $\Delta G$ $[\mathrm{kJ} / \mathrm{mol}]$ against $[\mathrm{GPa}]$ for $\mathrm{YN}$.

Input data and model parameters of YN.

TABLE I

\begin{tabular}{c|c|c|c|c|c}
\hline \hline Compound & \multicolumn{2}{|c|}{ Input data } & \multicolumn{3}{c}{ Model parameter } \\
\hline & $r[\AA]$ & $U[\mathrm{~kJ} / \mathrm{mol}]$ & $b\left[10^{-21} \mathrm{~J}\right]$ & $\rho[\AA]$ & $f(r)$ \\
\hline $\mathrm{YN}$ & 2.4000 & 131.1000 & 05.1530 & 0.4000 & -0.1412
\end{tabular}

TABLE II

Calculated transition pressures in GPa, associated volume collapses, elastic constants and their combination in $\mathrm{GPa}$.

\begin{tabular}{c|c|c|c|c}
\hline \hline \multirow{2}{*}{ Compound } & \multirow{2}{*}{ Transition } & \multicolumn{2}{|c|}{ Transition pressure [GPa] } & Volume collapses \\
\cline { 3 - 5 } & & present & others & present \\
\hline YN & $\mathrm{B}_{1} \rightarrow \mathrm{B}_{2}$ & 140 & $(138)^{a}$ & $10 \%$ \\
\hline
\end{tabular}

a Ref. [6].

In addition we have calculated the relative volume $[V(P) / V(0)]$ to obtain the equation of state for $\mathrm{B}_{1}$ and $\mathrm{B}_{2}$ phases for $\mathrm{YN}$ associated with various compressions and plotted them against pressures $(P)$ to depict the phase diagram in Fig. 2. The volume collapse $\left[\Delta V\left(P_{t}\right) / V(0)\right]$ accompanied by phase transition has been obtained from this figure and the values of volume collapse $-\Delta V\left(P_{t}\right) / V(0)$ in percentage, have been presented in Table II. A look at Table II reveals that density functional theory with full potential linearized augmented plane wave (FP-LAPW) shows a $\mathrm{B}_{1}-\mathrm{B}_{2}$ transition at $138 \mathrm{GPa}$ while our value $(140 \mathrm{GPa})$ is near to this value. It is also seen from Fig. 2 and Table II that the relative volume compression for $\mathrm{YN}$ is $10 \%$.

Our calculated value of the bulk modulus as shown in Table III is $155 \mathrm{GPa}$ and this value is compared to available computed data and it is closer to results of Mancera [6] calculated by first principle calculation than other data by using FLAPW and FLAPW-generalized gradient approximation (FLAPW-GGA) [8]. The study of second order elastic constants and their combinations are also important to know as they provide information about the interatomic and elastic behavior of these compounds. We have computed the second-order elastic constants (SOEC's) and their combinations, which are shown in Table III. The quantities $C_{S}$ and $C_{44}$ are the shear and tetragonal moduli of a cubic crystal. It is necessary for lattice to satisfy Born criterion for mechanically stable state. For this purpose, the elastic energy density must be a positive definite quadratic function of strain. In order to fulfill the above criterion the principal minor (eigenvalues) of the elastic constant matrix should all be positive. TBPM model confirmed that the features of these compounds show the same trends as earlier reported for pressure dependence of elastic stiffness for $\mathrm{PbTe}$ and $\mathrm{SnTe}$ [23] possessing the $\mathrm{NaCl}$ structure with $\mathrm{B}_{1}$ and $\mathrm{B}_{2}$ phase transitions. Vukcevich [24] also stated the high pressure stability criterion for ionic crystal, the stable phase of a crystal is one in which the shear elastic constant $C_{44}$ is non-zero (for mechanical stability) 


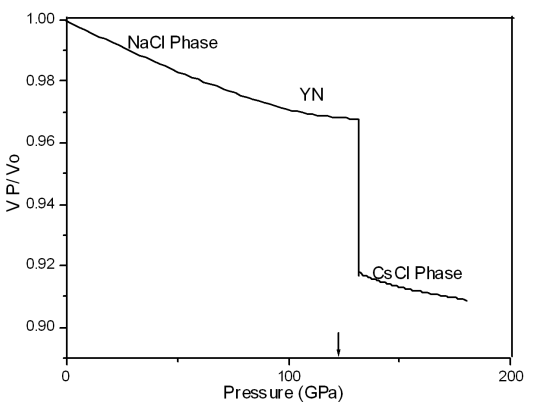

Fig. 2. Variation of relative volume $V(P) / V(0)$ with pressure $P[\mathrm{GPa}]$, present values of phase transition pressures are shown by solid and others by arrow for $\mathrm{YN}$.

TABLE III

The calculated values of second order elastic constants (SOECs), their combinations and third order elastic constants (TOECs) of YN.

\begin{tabular}{c|c|c}
\hline \hline ECs [GPa] & YN & Ref. \\
\hline$B_{T}$ & 155 & present \\
& $157^{a}$ & others \\
& $204^{b}$ & FLAPW \\
& $163^{c}$ & FLAPW-GGA \\
$C_{S}$ & 890 & present \\
$C_{11}$ & 188 & present \\
$C_{12}$ & 175 & present \\
$C_{44}$ & 253 & present \\
$C_{L}$ & 432 & present \\
$C_{111}$ & -8.65 & present \\
$C_{112}$ & -8.44 & present \\
$C_{166}$ & -0.26 & present \\
$C_{123}$ & 9.21 & present \\
$C_{144}$ & 1.86 & present \\
$C_{456}$ & 0.66 & present \\
\hline
\end{tabular}

${ }^{a}$ Ref. [6]; ${ }^{b}$ Ref. [8]; ${ }^{c}$ Ref. [8].

and which has the lowest potential energy among the mechanically stable lattices. Thus the stability of NaCl-type structure in terms of elastic constants should satisfy the following conditions

$$
\begin{aligned}
& S=\left(C_{11}-C_{12}\right) / 2>0, \quad B_{T}=\left(C_{11}+2 C_{12}\right) / 3>0, \\
& C_{44}>0 .
\end{aligned}
$$

In addition we have also calculated third order elastic constants (TOECs), although they are of only academic interest at present but they may be useful for experimentalists to work on present material. Due to lack of availability of experimental data on this material we could not provide a good support to our result, but the trends observed here are very similar to those observed by other workers [25] with the same structure reporting $\mathrm{B}_{1}-\mathrm{B}_{2}$ phase transition. Also it is found from Table III that our calculated values of TOECs and Debye temperature are in good agreement with available data [3]. It is clear from Fig. 3 that $C_{S}=\left(C_{11}-C_{12}\right) / 2$ with pressure decreases slightly but does not approach zero at phase transition pressure and this fulfils one of the conditions given by Eq. (15). It is clear from Table IV that the pressure derivative of bulk modulus and Debye temperature are in close range with other theoretical results where pressure decreases slightly but cannot approach zero at phase transition pressure. Musgrave and Pople [26] pointed out that if either $C_{44}$ or $C_{S}=\left(C_{11}-C_{12}\right) / 2$ go to zero as the pressure is enhanced, then the structure is unstable and the crystal transforms by a spontaneous shear. The values of the $\mathrm{d} C_{S} / \mathrm{d} P$ and $\mathrm{d} C_{44} / \mathrm{d} P$ also increase with increasing pressure. The value of $\mathrm{d} B_{T} / \mathrm{d} P$ is matching the value reported by others. Here we hope that the present calculation, which is mostly related with the basic understanding of the physics related with the interactions, will stimulate further work in this area and increase the interest of the scientific community in this material.

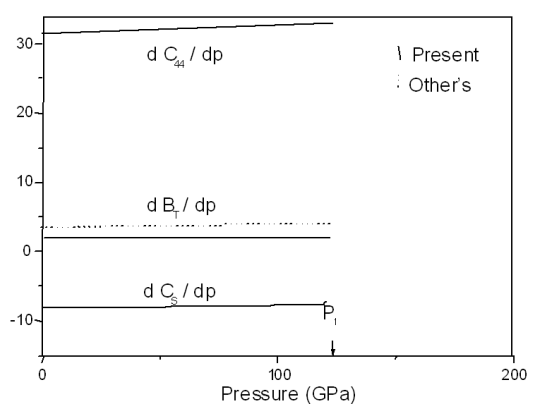

Fig. 3. Variation of pressure derivative of tetragonal modulus $\mathrm{d} C_{44} / \mathrm{d} P, \mathrm{~d} B_{T} / \mathrm{d} P$ shear modulus and $\mathrm{d} C_{S} / \mathrm{d} P$, with pressure for YN. The solid curve shows the present values of $\mathrm{YN}$ up to phase transition pressure $\left(P_{\mathrm{t}}\right)$, respectively.

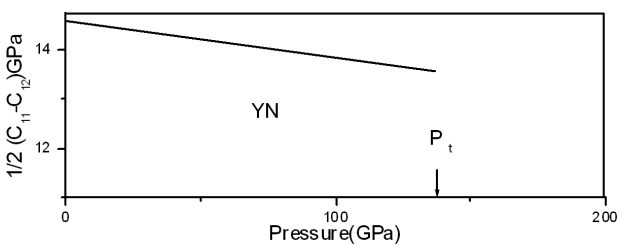

Fig. 4. Shear moduli $\left(C_{11}-C_{12}\right) / 2$ are plotted against pressure $P[\mathrm{GPa}]$ for $\mathrm{YN}$.

The success achieved in the present investigation can be ascribed to the inclusion of the charge transfer (or three-body) effects which seems to be of great importance at high pressure when the inter-ionic potential reduces considerably and the coordination number increases. Here, it is noted that our calculated values of phase transition pressure is in better agreement as compared to available theoretical data. 
TABLE IV

Pressure derivatives of SOEC's and Debye temperature $\left(\Theta_{\mathrm{D}}\right)$ of $\mathrm{YN}$.

\begin{tabular}{c|c|c}
\hline \hline Properties & YN & References \\
\hline Debye $[\mathrm{K}]$ & 607 & present \\
temperature & $595^{a}$ & others \\
\hline $\mathrm{d} C_{S} / \mathrm{d} P$ & -8.42 & present \\
$\mathrm{d} C_{44} / \mathrm{d} P$ & 30.84 & present \\
$\mathrm{d} B_{T} / \mathrm{d} P$ & 3.10 & present \\
& $3.50^{a}$ & others \\
\hline
\end{tabular}

${ }^{a}$ Ref. [3].

\section{Conclusions}

In summary, we have developed and applied three-body potential approach to investigate the structural, elastic and stability conditions of YN. The results are summarized as follows:

1. The $\mathrm{YN}$ at ambient conditions crystallizes in $\mathrm{NaCl}\left(\mathrm{B}_{1}\right)$ structure and under pressure shows a $\mathrm{B}_{1}$ to $\mathrm{B}_{2}$ phase transition. Our results with present approach including TBI effect are showing good matching with available results.

2. Our model is able to predict the stability of the phase $\mathrm{B}_{1}$ correctly as the value of $\Delta G$ is positive at zero pressure.

3. During the phase transition from $\mathrm{NaCl}\left(\mathrm{B}_{1}\right)$ to $\mathrm{CsCl}\left(\mathrm{B}_{2}\right)$ the volume discontinuity in pressure volume phase diagram identifies the occurrence of first-order phase transition.

4. The stability criterion under pressure which is important tool for geologists to work is studied successfully.

5. We have also shown probably for the first time the effect of high pressure on the pressure derivatives of second-order elastic constants.

6. We have calculated third order elastic constants and the Debye temperature for the first time.

From the overall study we may conclude that the present three-body potential approach is fairly adequate for the prediction of high pressure $B_{1}-B_{2}$ phase transition, associated volume collapses, bulk modulus, second order elastic constants, the Debye temperature, third order elastic constants, pressure derivatives of shear modulus $\mathrm{d} C_{S} / \mathrm{d} P$, tetragonal modulus $\mathrm{d} C_{44} / \mathrm{d} P$ and $\left(C_{11}-C_{12}\right) / 2$. Also the stability criterion in $\mathrm{YN}$ has been checked successfully.

\section{Acknowledgments}

One of the authors (S.S.) is thankful to BRNS, Mumbai for financial support.

\section{References}

[1] R. Ahuja, O. Eriksson, Phys. Rev. B 53, 3072 (1996).

[2] T. Eto, S. Endo, M. Imai, Y. Katayama, T. Kikegawa, Phys. Rev. B 6, 114984 (2000).

[3] A. Fernandez, J. Guilermet Hugland, G. Grimvall, Phys. Rev. B 45, 11557 (1992).

[4] V.A. Gubanov, A.L. Ianovsky, V.P. Zhukov, Electronic Structure of Refractory Carbides and Nitrides, Cambridge University Press, Cambridge 1994.

[5] H.A. Al-Brithen, A.R. Smith, D. Gall, Phys. Rev. B 70, 045303 (2004).

[6] L. Mancera, J.A. Rodriguez, N. Takeuchi, J. Phys. Condens. Matter 15, 2625 (2003).

[7] D. Gall, M. Städele, K. Järrendahl, I. Petrov, P. Desjardins, R.T. Haasch, T.-Y. Lee, J.E. Greene, Phys. Rev. B 63, 125119 (2001).

[8] C. Stampfl, W. Mannstadt, R. Asahi, A.J. Freeman, Phys. Rev. B 63, 155106 (2001).

[9] C. Stampfl, R. Asahi, A.J. Freeman, Phys. Rev. B 65, 161204 (2002).

[10] T. Liapina, A. Leineweber, J.E. Mittemeijer, W. Kockelmann, Acta Mater. 52, 173 (2004).

[11] H.S. Jhi, G.S. Louie, L.M. Cohen J. Ihm, Phys. Rev. Lett. 86, 3348 (2001).

[12] E.R. Rundle, Acta Crystallogr. 1, 180 (1948).

[13] A. Leineweber, H. Jacobs, J. Alloys Comp. 308, 178 (2000).

[14] J. Haglund, G. Grimvall, T. Jarlborg, A. Fernandez, A.F. Guillermet, Phys. Rev. B 43, 14400 (1991).

[15] N. Takeuchi, Phys. Rev. B 66, 153405 (2002).

[16] R.K. Singh, Phys. Rep. 85, 262 (1982).

[17] R.K. Singh, S. Singh, Phys. Rev. B 39, 671 (1989); Phys. Rev. B 45, 1019 (1992).

[18] U.C. Sharma, M.P. Verma, Phys. Status Solidi B 102, 487 (1980).

[19] S. Singh, R.K. Singh, High Pressure Res. J. 21, 105 (2001).

[20] J. Shanker, V.C. Jain, J.P. Singh Phys. Rev. B 22, 1083 (1986).

[21] J. Shanker, M. Kumar, Phys. Status Solidi B $\mathbf{1 7 9}$ 351 (1993).

[22] S. Singh, J. Phys. Soc. Jpn. 71, 2477 (2002).

[23] A.J. Miller, G.A. Saunders, Y.K. Yogurtev, J. Phys. C 14, 1569 (1981).

[24] M.R. Vukcevich, Phys. Status Solidi B 54, 435 (1972).

[25] M.J.L. Sangster, U. Schroder, R.M. Atwood, J. Phys. C 11, 1523 (1978).

[26] M.J.P. Musgrave, J.L. Pople, J. Phys. Chem. Solids 23, 323 (1962). 